\title{
Exogenous regucalcin suppresses the growth of human liver cancer HepG2 cells in vitro
}

\author{
MASAYOSHI YAMAGUCHI $^{1}$ and TOMIYASU MURATA ${ }^{2}$ \\ ${ }^{1}$ Department of Pathology and Laboratory Medicine, David Geffen School of Medicine, University of California (UCLA), \\ Los Angeles, CA 90095-1781, USA; ${ }^{2}$ Laboratory of Analytical Neurobiology, \\ Faculty of Pharmacy, Meijo University, Nagoya 468-8503, Japan
}

Received November 11, 2017; Accepted March 28, 2018

DOI: $10.3892 /$ or.2018.6357

\begin{abstract}
Regucalcin gene ( $r g n$ ) is localized on the X chromosome and it plays a pivotal role as a regulatory protein in the intracellular signaling process implicated in the transcription activity of manifold cells. The expression of rgn was demonstrated to be suppressed in tumor tissues of human subjects, indicating a potential role as a suppressor of tumorigenesis. Regucalcin, which is produced from tissues including liver, is released in the serum of human subjects and animals. The role of serum regucalcin has been poorly understood. To determine the effects of exogenous regucalcin on modeled human liver cancer HepG 2 cells, the cells were cultured in the presence of exogenous regucalcin in vitro. The proliferation of HepG2 cells was suppressed after being cultured with the addition of regucalcin $(0.01-10 \mathrm{nM})$ into the culture medium. The addition of regucalcin did not exhibit effects on apoptotic cell death in HepG2 cells in vitro. Suppresive effects of exogenous regucalcin on cell proliferation were not enhanced in culture with various signaling inhibitors including tumor necrosis factor- $\alpha$, Bay K 8644, PD98059, staurosporine, worthomannin, 5,6-dichloro-1- $\beta$-D-ribofuranosylbenzimidazole or gemcitabine, which were found to suppress cell proliferation. In addition, exogenous regucalcin suppressed the formation of colonies of cultured HepG2 cells in vitro. The present study demonstrated that exogenous regucalcin exhibited an inhibitory effect on the growth of human liver cancer cells, thus proposing a gene therapy strategy for cancer treatment.
\end{abstract}

Correspondence to: Dr Masayoshi Yamaguchi, Department of Pathology and Laboratory Medicine, David Geffen School of Medicine, University of California, Los Angeles (UCLA), 700 Tiverton Avenue, Los Angeles, CA 90095-1781, USA

E-mail: yamamasa11555@yahoo.co.jp

Key words: regucalcin, cell proliferation, liver cancer, HepG2 cells, cell signaling, carcinogenesis

\section{Introduction}

Regucalcin was originally found as a novel calcium-binding protein in 1978 (1-4). This protein plays a manifold role as a suppressor of various signaling processes in the regulation of cellular function in various types of cells and tissues (5-7). The regucalcin gene ( $r g n)$ is localized on the $\mathrm{X}$ chromosome and is identified in over 15 species consisting of regucalcin family in vertebrate and invertebrate species (7-10). The expression of $r g n$ was regulated by the activity of various physiological factors including peptide and steroid hormones as well as cytokines $(11,12)$ and the process of this gene expression was related to various transcription factors including AP-1, NF1-A1, RGPR-p117, $\beta$-catenin and other factors (12). This process was implicated in the phosphorylation and dephosphorylation of various intracellular signaling factors in the cytoplasm and nucleus in vitro (12). Regucalcin was largely present in the cytoplasm, and was translocated into the nucleus through mechanisms which depend on the activation of calcium signaling related to protein kinase $\mathrm{C}$ in cells. In addition, nuclear regucalcin regulated transcription activity (13). Regucalcin exerted multifunctional effects in maintaining cellular calcium homeostasis, inhibition of manifold protein kinases, protein phosphatases and protein synthesis in the cytoplasm and nucleus, and nuclear function in various types of cells $(5-7,13)$. Notably, regucalcin has been demonstrated to inhibit cell proliferation and apoptotic cell death, which were mediated through the stimulation of signaling factors $(14,15)$. Accumulating evidence indicated that regucalcin played a pivotal role in maintaining cell homeostasis as a modulator protein in the cell signaling process implicated in transcription activity $(14,15)$.

Furthermore, regucalcin has been demonstrated to play a pathophysiological role in metabolic disorders (16-19). Notably, regucalcin played a crucial role as a suppressor in cell proliferation and carcinogenesis $(14,19)$. Endogenous regucalcin was demonstrated to suppress cell proliferation of cloned-rat normal kidney NRK52E cells (20) and rat hepatoma H4-II-E cells in vitro (21) due to inducing G1 and G2/M phase cell cycle arrest $(20,21)$. Mechanically, the suppressive effects of overexpressed endogenous regucalcin on cell proliferation were shown to be mediated through the suppression of the activities of various protein kinases, protein phosphatases and 
PI3 kinase implicated in various signaling pathways $(14,19)$. Furthermore, endogenous regucalcin has been observed to enhance the expression of $p 53$ and $R b$ mRNAs (22), tumorsuppressor genes, and suppress the expression of $\mathrm{c}-M y c$, Ha-ras, c-jun and Chk2 mRNAs (23), enhancer genes of tumorigenesis in hepatoma cells $(13,19,24)$. In addition, regucalcin was revealed to inhibit cytoplasmic protein synthesis in the cytoplasm and DNA and RNA synthesis in the nucleus of liver and hepatoma cells $(13,19,24)$. Thus, endogenous regucalcin was demonstrated to inhibit cell proliferation implicated in multifunctional pathways in cancer cells $(17,19)$.

Notably, the expression of $r g n$ and its protein levels were downregulated in tumor tissues of human subjects and cancer cells $(19,25)$. Survival rates were demonstrated to be prolonged in patients with pancreatic, breast, liver and lung cancers with increased $r g n$ expression (26-29). Overexpressed endogenous regucalcin was revealed to suppress the proliferation of human pancreatic cancer MiaPaCa-2 (26), MDA-MB-231 human breast cancer (27), liver cancer HepG2 (28) and human lung adenocarcinoma A549 cells (28) in vitro. Regucalcin has been proposed to reveal a potential activity as a suppressor of human carcinogenesis.

Regucalcin, which is produced from the tissues including liver, is present in the serum of human subjects and animals $(18,30)$. Extracellular regucalcin may play a part in the regulation of cell function. However, this has been poorly understood. The aim of the present study was to investigate whether exogenous regucalcin revealed a suppressive effect on the growth of human liver cancer cells. We observed that exogenous regucalcin suppressed the growth of human liver cancer HepG2 cells in vitro.

\section{Materials and methods}

Materials. The $\alpha$-minimum essential medium ( $\alpha$-MEM; with glutamine) with antibiotics (penicillin and streptomycin) were purchased from Gibco Life Technologies Corporation (Grand Island, NY, USA). Fetal bovine serum (FBS) was obtained from Omega Scientific Inc. (Tarzana, CA, USA). Tumor necrosis factor- $\alpha$ (TNF- $\alpha$ ) was obtained from R\&D Systems (Minneapolis, MN, USA). PD98059, staurosporine, Bay K 8644, worthomannin or 5, 6-dichloro-1- $\beta$-D-ribofuranosylbenzimidazole (DRB), crystal violet and all other reagents were purchased from Sigma-Aldrich (St. Louis, MO, USA) unless otherwise specified. Gemcitabine was obtained from Hospira, Inc. (Lake Forest, IL, USA) and it was diluted in phosphate-buffered saline (PBS; Sigma-Aldrich).

Regucalcin. Regucalcin was isolated from rat liver cytosol as previously described (1). Rat livers were perfused with Tris-HCl buffer (pH 7.4), containing $100 \mathrm{mM}$ Tris, $120 \mathrm{mM}$ $\mathrm{NaCl}, 4 \mathrm{mM} \mathrm{KCl}$, cooled at $4^{\circ} \mathrm{C}$ to remove blood. Subsequently, the livers were immediately removed, cut into small pieces, suspended 1:4 (weight/volume) in Tris- $\mathrm{HCl}$ buffer ( $\mathrm{pH} 7.4)$ and homogenized in a Potter-Elvehjem homogenizer (Takashima Corporation, Tokyo, Japan) with a Teflon pestle (Thomas Scientific, Swedesboro, NJ, USA) with cooling at $4^{\circ} \mathrm{C}$ (1). The homogenate was spun at $5,500 \mathrm{x} \mathrm{g}$ in a refrigerated centrifuge for $10 \mathrm{~min}$, and the supernatant was spun at 105,00 x $\mathrm{g}$ for $60 \mathrm{~min}$ at $4^{\circ} \mathrm{C}$. The resulting supernatant was isolated to electorophoretic homogeneity by gel filtration on Sephadex G-75 and G-50 (Santa Cruz Biotechnology, Dallas, TX, USA), followed by ion-exchange chromatography on diethylaminoethyl (DEAE)-cellulose as previously described (1). The purity of the isolated regucalcin was confirmed using SDS-gel electrophoresis and western blot analysis (1). Isolated regucalcin was used in the following experiments.

Human liver cancer cells. We used human hepatoblastoma liver cancer HepG2 cells which were obtained from the American Type Culture Collection (Rockville, MD, USA). The HepG2 cell line was derived from a 15-year-old child with primary hepatoblastoma (31), and its derivative C3A was not from hepatocellular carcinoma $(31,32)$.

Cell proliferation. HepG2 cells $\left(1 \times 10^{5} / \mathrm{ml}\right.$ per well) were cultured using a 24-well plate in $\alpha$-MEM (containing $10 \% \mathrm{FBS}, 1 \%$ penicillin plus streptomycin, and $1 \%$ fungizone) in the presence or absence of regucalcin $(0.01,0.1,0.5,1$ or $10 \mathrm{nM}$ ) for 1, 2, 3 and 6 days $(20,21)$. In separate experiments, cells $\left(1 \times 10^{5} / \mathrm{ml}\right.$ per well) were cultured for 3 days in DMEM containing $10 \% \mathrm{FBS}$ and $1 \% \mathrm{P} / \mathrm{S}$ in the presence of TNF- $\alpha$ (1 ng/ml), Bay K $8644(1 \mu \mathrm{M})$, PD98059 $(1 \mu \mathrm{M})$, staurosporin $(0.1 \mu \mathrm{M})$, worthomannin $(1 \mu \mathrm{M})$, DRB $(1 \mu \mathrm{M})$ or gemcitabine $(10 \mathrm{nM})$, which were at an effective concentration. After the culture, the cells on dishes were detached to determine the cell number.

Cell death. HepG2 cells (1x105/ml per well) were cultured using a 24 -well plate in $\alpha$-MEM (containing $10 \% \mathrm{FBS}$, $1 \%$ penicillin plus streptomycin, and $1 \%$ fungizone) in the absence of regucalcin for 3 days to reach subconfluence, and then the cells were cultured in the presence or absence of regucalcin $(0.01,0.1,0.5,1$ or $10 \mathrm{nM})$ with or without gemcitabine (10 $\mathrm{nM})$ for 24 or $48 \mathrm{~h}$ (15). After the culture, the cells on dishes were detached to determine the cell number.

Cell counting. Following trypsinization of each of culture dishes using $0.05 \%$ trypsin plus EDTA in $\mathrm{Ca}^{2+} / \mathrm{Mg}^{2+}$-free PBS for $2 \mathrm{~min}$ at $37^{\circ} \mathrm{C}$, cells attached on dishes were collected by pipetting $(20,21)$. The cells were suspended on PBS solution and stained with eosin. Cell numbers were counted under a microscope (Nikon TMS; Nikon, Tokyo, Japan) using a hemocytometer plate (Sigma-Aldrich). We took the average of two countings for each dish. Cell number is shown as the number per well of each plate.

Colony formation assay. HepG2 cells were seeded into 6-well dishes at a density of $1 \times 10^{3} /$ well and cultured in medium containing $10 \% \mathrm{FBS}$ under $5 \% \mathrm{CO}_{2}$ at $37^{\circ} \mathrm{C}$ for 14 days, when visible clones were formed on the plates (33). Obtained colonies were washed with PBS ( $2 \mathrm{ml}, 3$ times) and fixed with methanol $(0.5 \mathrm{ml} /$ well $)$ for $20 \mathrm{~min}$ at room temperature, and then washed 3 times with PBS. Subsequently, the colonies were stained with $0.1 \%$ crystal violet $(1 \mathrm{ml})$ for $30 \mathrm{~min}$ at room temperature. Stained cells were washed 4 times with PBS $(2 \mathrm{ml})$. The plate was air-dried for $2 \mathrm{~h}$ at room temperature. The colony containing more than 50 cells was counted under a microscope (Nikon TMS; Nikon). 

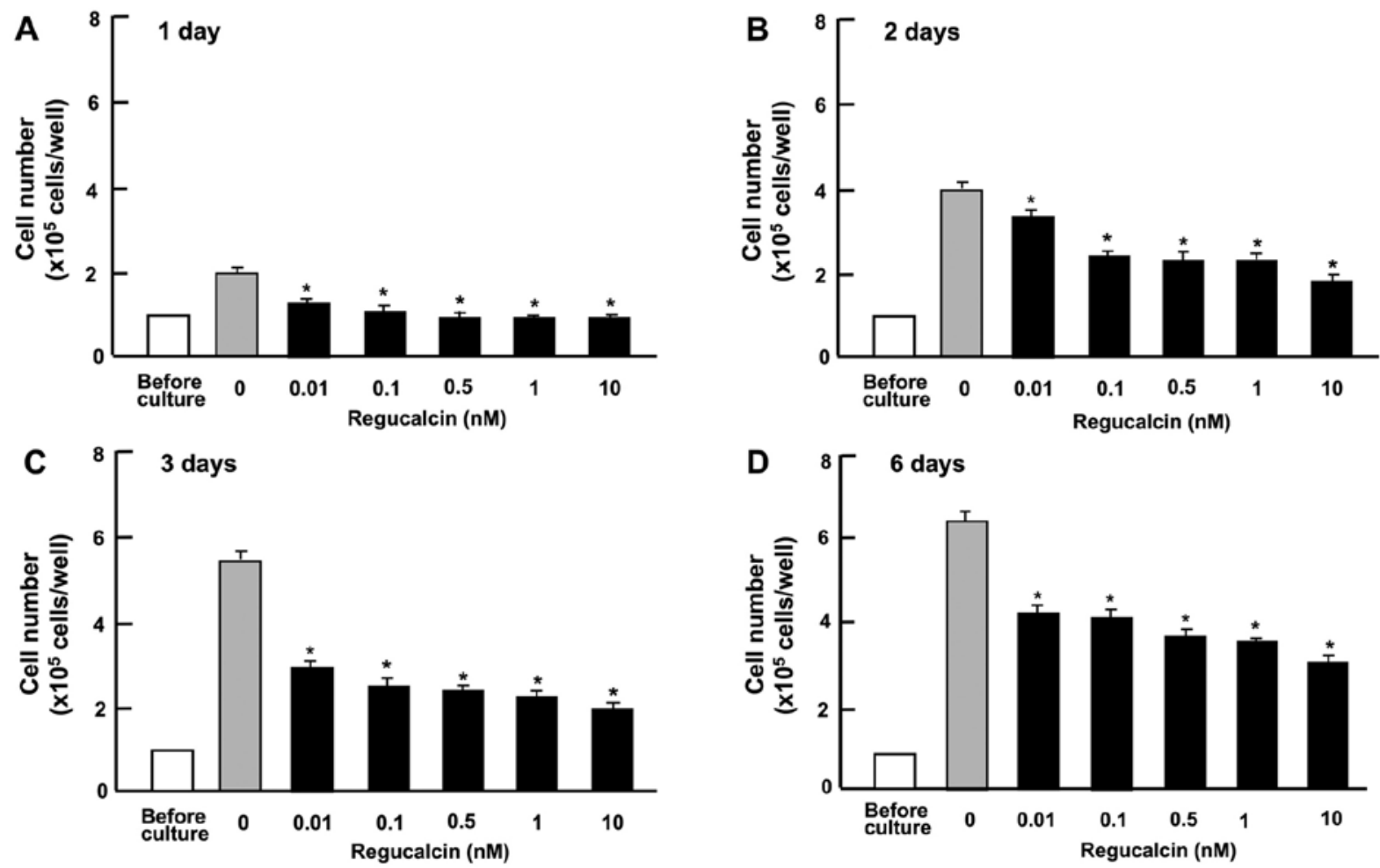

Figure 1. Exogenous regucalcin suppresses the proliferation of human liver cancer HepG2 cells in vitro. Cells were cultured in $\alpha$-MEM (containing $10 \%$ FBS, $1 \%$ penicillin, streptomycin, and fungizone) after the addition of either vehicle (PBS) or exogenous regucalcin (0.01-10 nM) for (A) 1 , (B) 2 , (C) 3 or (D) 6 days. After the culture, the number of cells attached on dishes was counted. Data are presented as the mean \pm SD obtained from 8 wells of 2 replicate plates per dataset using different dishes and cell preparations. "P<0.001 vs. control (grey bar). One-way ANOVA, Tukey-Kramer post test.
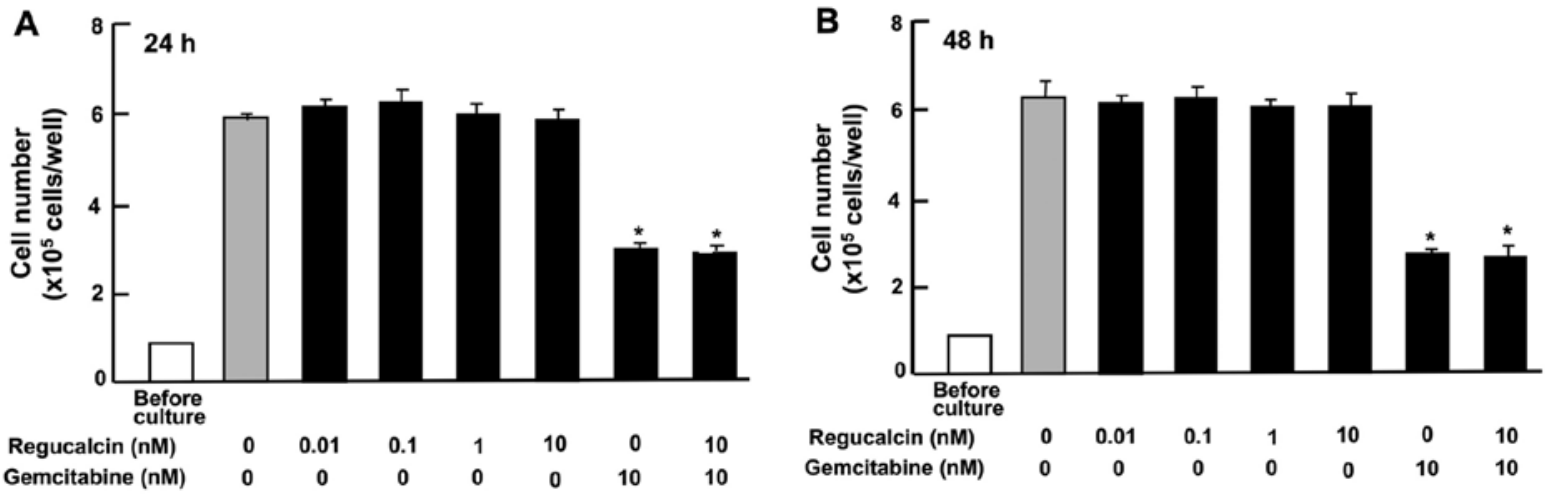

Figure 2. Effect of exogenous regucalcin on the death of human liver cancer HepG2 cells in vitro. Cells were cultured for 3 days upon reaching subconfluency, and then the cells were cultured for (A) $24 \mathrm{~h}$ or (B) $48 \mathrm{~h}$, after the addition of either vehicle (PBS) or regucalcin $(0.01,0.1,0.5,1$ or $10 \mathrm{nM})$ with or without gemcitabine $(10 \mathrm{nM})$. After the culture, the number of cells attached on the dishes was counted. Data are presented as the mean \pm SD obtained from 8 wells of 2 replicate plates per dataset using different dishes and cell preparations. " $\mathrm{P}<0.001$ vs. the control (grey bar). One-way ANOVA, Tukey-Kramer post test.

Statistical analysis. Statistical significance was evaluated using GraphPad InStat version 3 for Windows XP (GraphPad Software Inc., La Jolla, CA, USA). Multiple comparisons were performed using one-way analysis of variance (ANOVA) with Tukey-Kramer multiple comparisons post test for indicated parametric data. $\mathrm{P}<0.05$ was considered to indicate a statistically significant difference.

\section{Results}

In the present study we examined whether suppressive effects of exogenous regucalcin on cell proliferation were revealed in human hepatoblastoma livercancer HepG2 cells in vitro. HepG2 cells were cultured with the addition of either vehicle (PBS) or exogenous regucalcin (0.01-10 $\mathrm{nM}$ ) for 1-6 days (Fig. 1). An increasing of culture periods raised cell number in the control group. Culture with the addition of exogenous regucalcin suppressed the elevation of cell numbers (Fig. 1), revealing that the proliferation of HepG2 cells was suppressed with the physiological levels of regucalcin which is present in the serum $(18,30)$.

The effect of exogenous regucalcin on the death of HepG2 cells in vitro is displayed in Fig. 2. Cells were cultured for 3 days upon reaching subconfluency, and then 


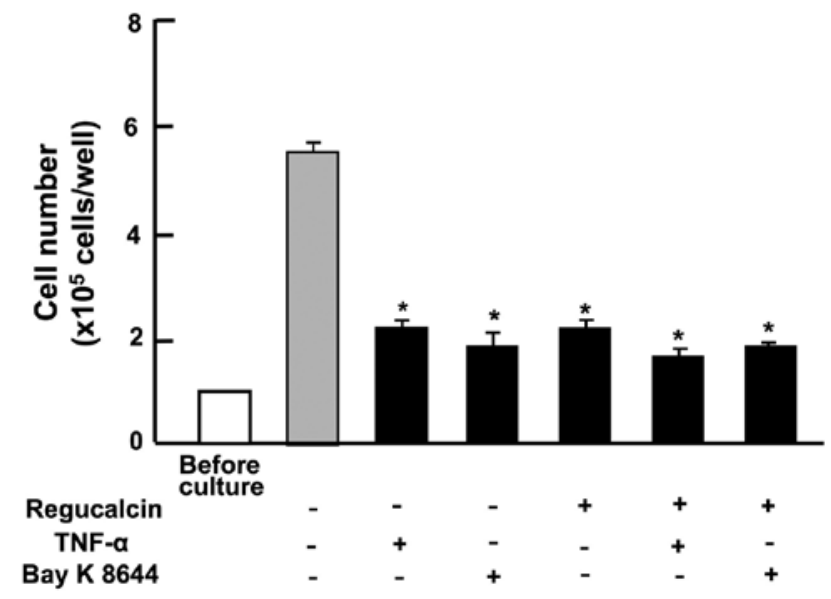

Figure 3. Suppressive effects of regucalcin on the proliferation of human liver cancer HepG2 cells are not potentiated in the presence of TNF- $\alpha$ or Bay K 8644 in vitro. Cells were cultured for 3 days in the presence of either vehicle (PBS) or exogenous regucalcin $(10 \mathrm{nM})$ with or without the addition of TNF- $\alpha(1 \mathrm{ng} / \mathrm{ml})$ or Bay K $8644(1 \mu \mathrm{M})$. After the culture, the number of cells attached on dishes was counted. Data are presented as the mean \pm SD obtained from 8 wells of 2 replicate plates per dataset using different dishes and cell preparations. ${ }^{*} \mathrm{P}<0.001$ vs. the control (grey bar). One-way ANOVA, Tukey-Kramer post test.

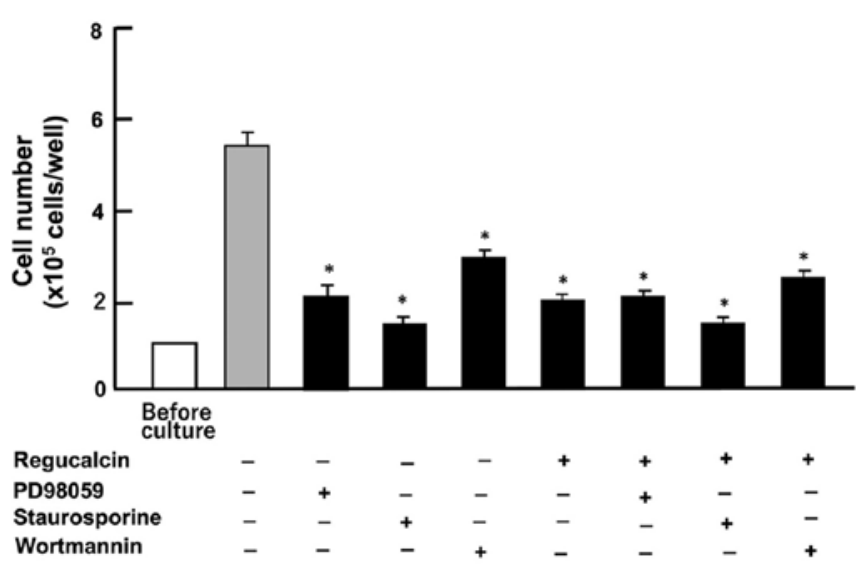

Figure 4. Suppressive effects of regucalcin on the proliferation of human liver cancer HepG2 cells in the presence of various intracellular signaling inhibitors in vitro. Cells were cultured for 3 days after the addition of either vehicle (0.1\% ethanol) or regucalcin $(10 \mathrm{nM})$ with or without PD98059 $(1 \mu \mathrm{M})$, staurosporine $(0.1 \mu \mathrm{M})$ or wortmannin $(1 \mu \mathrm{M})$ at an effective concentration. After the culture, the number of cells attached on dishes was counted. Data are presented as the mean $\pm \mathrm{SD}$ obtained from 8 wells of 2 replicate plates per dataset using different dishes and cell preparations. "P<0.001 vs. the control (grey bar). One-way ANOVA, Tukey-Kramer post test.

the cells were cultured for 24 or $48 \mathrm{~h}$, after the addition of either vehicle (PBS) or regucalcin (0.01-10 nM) with or without gemcitabine $(10 \mathrm{nM})$. The number of HepG2 cells was not changed in the presence of exogenous regucalcin, although the addition of gemcitabine caused the death of cells (Fig. 2). Thus, exogenous regucalcin exhibited a suppressive effect on cell proliferation independently of the death of HepG2 cells.

The suppressive effects of exogenous regucalcin on the proliferation of HepG2 cells were compared with the effects of other factors that inhibit cell growth (Fig. 3). The effects of exogenous regucalcin $(10 \mathrm{nM})$ suppressing

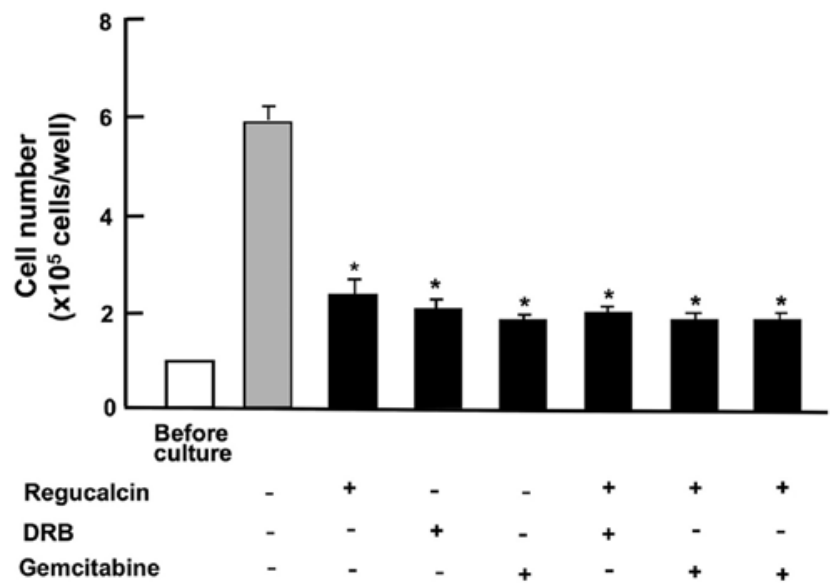

Figure 5. Effects of regucalcin in inhibiting the proliferation of human liver cancer HepG2 cells with or without DRB or gemcitabine that influence transcription activities in vitro. Cells were cultured for 3 days after the addition of either vehicle $(0.1 \%$ ethanol) or regucalcin $(10 \mathrm{nM})$ with or without DRB $(1 \mu \mathrm{M})$ or gemcitabine $(10 \mathrm{nM})$ at an effective concentration. After culture, the number of cells attached on dishes was counted. Data are presented as the mean \pm SD obtained from 8 wells of 2 replicate plates per dataset using different dishes and cell preparations. ${ }^{*} \mathrm{P}<0.001$ vs. the control (grey bar). One-way ANOVA, Tukey-Kramer post test.

the proliferation of HepG2 cells were not potentiated by the addition of TNF- $\alpha(1 \mathrm{ng} / \mathrm{ml})$, an inducer of nuclear factor- $\kappa \mathrm{B}(\mathrm{NF}-\kappa \mathrm{B})$ signaling (34), or Bay $\mathrm{K} 8644(1 \mu \mathrm{M})$, an agonist of $\mathrm{Ca}^{2+}$ entry into cells (35), that caused a decrease in the number of cells (Fig. 3).

Subsequently, we determined whether the suppressive effects of exogenous regucalcin on the proliferation of HepG2 cells were implicated in intracellular signaling pathways. The effects of exogenous regucalcin in suppressing cell proliferation were not enhanced by the addition of PD98059 $(1 \mu \mathrm{M})$, an extracellular signal-regulated kinase (ERK) inhibitor (36), staurosporin $(0.1 \mu \mathrm{M})$, an inhibitor of protein kinase $\mathrm{C}(37)$ and worthomannin $(1 \mu \mathrm{M})$, an inhibitor of phosphatidylinositol 3-kinase (PI3K) (38) (Fig. 4).

Subsequently, to determine whether the suppressive effects of exogenous regucalcin on the proliferation of HepG2 cells were implicated in nuclear function, we used DRB $(1 \mu \mathrm{M})$, an inhibitor of transcription activity with RNA polymerase II inhibition (39), or gemcitabine (10 nM), an antitumor drug that induces the damage of nuclear DNA (40). The suppressive effects of exogenous regucalcin on the proliferation of HepG2 cells were not altered by the addition of DRB or gemcitabine, which induced suppression of the proliferation of HepG2 cells (Fig. 5).

Furthermore, exogenous regucalcin was shown to decrease colony formation of HepG2 cells in vitro (Fig. 6). Culture with exogenous regucalcin (1 and $10 \mathrm{nM})$ led to a reduction of the number of colonies of HepG2 cells (Fig. 6). Thus, exogenous regucalcin was demonstrated to exhibit suppressive effects on colony formation due to inhibiting the proliferation of HepG2 cells in vitro.

\section{Discussion}

Regucalcin is produced from tissues including liver, and it has been shown to be present in the serum of human subjects and 

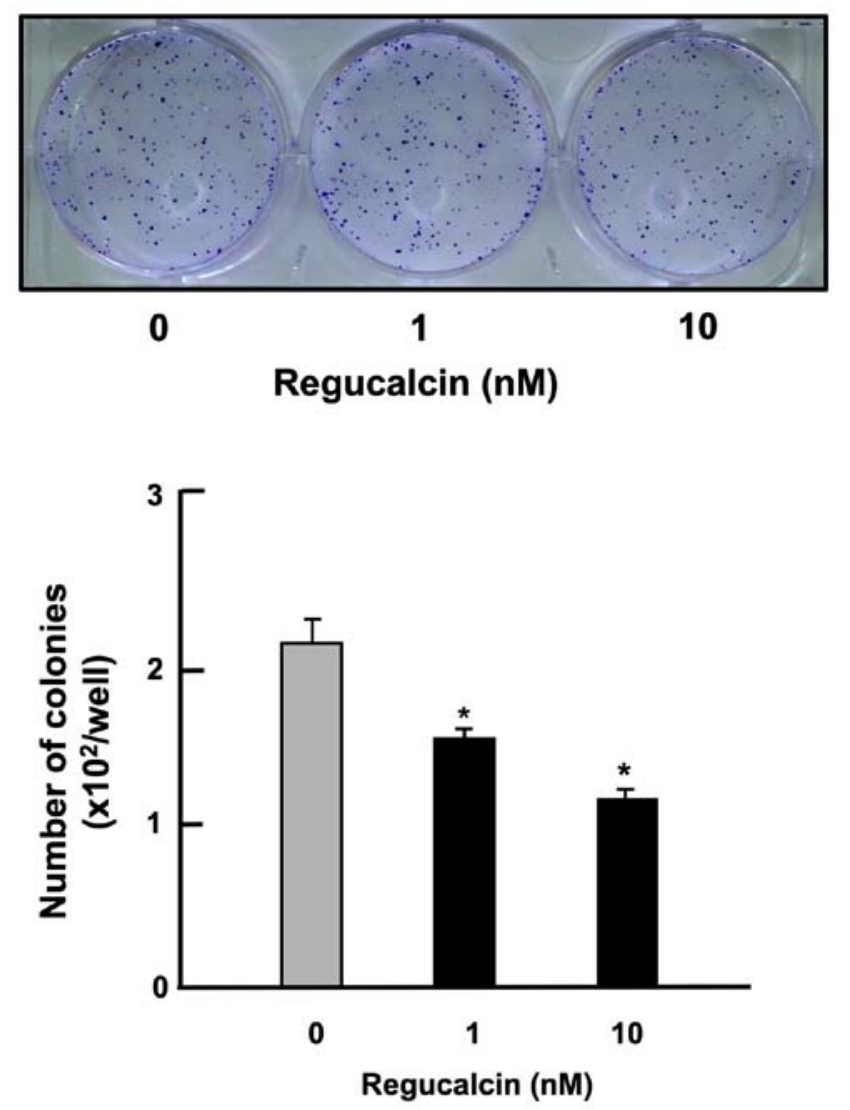

Figure 6. Exogenous regucalcin suppresses the colony formation of human liver cancer HepG2 cells in vitro. HepG2 cells were cultured with the addition of vehicle (PBS) or exogenous regucalcin (1 or $10 \mathrm{nM})$ for 14 days. After culture, colonies were stained with $0.5 \%$ crystal violet, and stained colonies were counted. The colony containing more than 50 cells was counted under a microscope. Data are presented as the mean \pm SD obtained from 8 wells of 2 replicate plates per dataset using different dishes and cell preparations. ${ }^{*} \mathrm{P}<0.001$ vs. the control (grey bar). One-way ANOVA, Tukey-Kramer post test.

animals $(18,30)$. Whether or not extracellular regucalcin is important in the regulation of cell function has been poorly understood. Exogenous regucalcin was demonstrated to bind the plasma membranes and activate plasma membrane $\left(\mathrm{Ca}^{2+}-\mathrm{Mg}^{2+}\right)$-adenosine triphosphatase in rat liver cells in vitro (41). Exogenous regucalcin may regulate the function of hepatoma cells. In the present study, we found that culture with exogenous regucalcin suppressed the growth in human hepatoblastoma liver cancer HepG2 cells, suggesting its role in the regulation of cell proliferation in vitro.

The effects of exogenous regucalcin in suppressing the proliferation of HepG 2 cells were not enhanced by the addition of TNF- $\alpha$, an enhancer of NF- $\mathrm{BB}$ signaling (34), Bay K 8644, an agonist of $\mathrm{Ca}^{2+}$ entry in the cells (35), staurosporin, an inhibitor of calcium-dependent protein kinase C (36), PD98059, an ERK inhibitor (37) and wortmannin, an inhibitor of PI3 kinase (38). The suppressive effects of exogenous regucalcin on the proliferation of HepG2 cells were not potentiated by the treatment of various inhibitors that regulate intracellular signaling pathways related to proliferation in vitro. Furthermore, the effects of exogenous regucalcin in suppressing cell proliferation were not potentiated in the presence of DRB, an inhibitor of transcription activity with RNA polymerase II inhibition (39). The effects of exogenous regucalcin in inhibiting the proliferation of HepG2 cells were implicated with various intracellular signaling processes. Exogenous regucalcin binds to the plasma membranes of HepG2 cells, and bound regucalcin may induce the generation of signaling factors that lead to the suppression of transcription activity-related signaling processes in the nucleus of HepG2 cells. In addition, it is hypothesized that exogenous regucalcin bound to liver plasma membranes may be internalized into hepatoma cells, and that internalized regucalcin demonstrates suppressive effects on the signaling pathways implicated to cell proliferation $(7,13,14)$. The exact mechanisms of action remain to be elucidated in further studies.

The effects of exogenous regucalcin in suppressing the proliferation of HepG2 cells were compared with that of gemcitabine, which is an antitumor drug, which causes nuclear DNA damage and apoptosis (40). Suppressive effects of exogenous regucalcin on the proliferation of HepG2 cells revealed similar effects with gemcitabine. Exogenous regucalcin did not cause the death of HepG2 cells in vitro, supporting the hypothesis that regucalcin does not possess an effect in inducing apoptotic cell death. Revealing the effects of exogenous regucalcin in inducing the proliferation of HepG2 cells was not based on apoptotic cell death. The mechanism by which exogenous regucalcin suppressed the proliferation of HepG2 cells may be based on different mode of action compared to that of gemcitabine. Exogenous regucalcin may be a useful tool to potentiate antitumor effects on human liver cancer cells in combination with gemcitabine.

Notably, culture with exogenous regucalcin was demonstrated to suppress colony formation of HepG2 cells in vitro. This effect may be based on exogenous regucalcin-induced suppression of the proliferation of HepG2 cells. Thus, exogenous regucalcin plays a suppressive role on the growth of human liver cancer cells. We used human hepatoblastoma liver cancer HepG2 cells in the present study. However, this is unlikely to affect our conclusions that exogenous regucalcin demonstrated suppressive effects on the colony formation and proliferation of liver cancer cells. In addition, it is possible that exogenous regucalcin demonstrated a suppressive effect on hepatocellular carcinoma and hepatoblastoma. However, this remains to be elucidated by using human hepatocellular carcinoma tumor cells.

Our previous studies demonstrated that exogenous regucalcin demonstrated suppressive effects on the proliferation of human pancreatic cancer MiaPaCa-2 cells (42) and MDA-MB-231 human breast cancer cells (43) in vitro. Furthermore, the present study revealed that exogenous regucalcin inhibited the growth of human liver cancer cells in vitro. Thus, exogenous regucalcin, which is produced in the tissues, may suppress the growth in various types of human cancer cells. Exogenous regucalcin has been suggested to contribute as a suppressor in the development of carcinogenesis, thus proposing a therapeutic strategy with regucalcin gene therapy.

\section{Acknowledgements}

The authors thank Dr Oliver Hankinson for his encouragement, David Geffen School of Medicine, University of California, California (UCLA). 


\section{Funding}

The present study was supported in part from the Foundation for Biomedical Research on Regucalcin, Japan.

\section{Availability of data and materials}

The datasets used during the present study are available from the corresponding author upon reasonable request.

\section{Authors' contributions}

MY conceived designed the study. MY and MT performed the experiment and discussed with TM. MY wrote the manuscript and MT reviewed and edited the manuscript. All authors read and approved the manuscript and agree to be accountable for all aspects of the research in ensuring that the accuracy or integrity of any part of the work are appropriately investigated and resolved.

\section{Ethics approval and consent to participate}

All experimental protocols consisted of cells cultured in vitro.

\section{Consent for publication}

Not applicable.

\section{Competing interests}

The authors state that they have no competing interests.

\section{References}

1. Yamaguchi M and Yamamoto T: Purification of calcium binding substance from soluble fraction of normal rat liver. Chem Pharm Bull 26: 1915-1918, 1978.

2. Yamaguchi M and Sakurai T: Inhibitory effect of calciumbinding protein regucalcin on $\mathrm{Ca}^{2+}$-activated DNA fragmentation in rat liver nuclei. FEBS Lett 279: 281-284, 1991.

3. Shimokawa $\mathrm{N}$ and Yamaguchi M: Molecular cloning and sequencing of the cDNA coding for a calcium-binding protein regucalcin from rat liver. FEBS Lett 327: 251-255, 1993.

4. Misawa $\mathrm{H}$ and Yamaguchi $\mathrm{M}$ : The gene of $\mathrm{Ca}^{2+}$-binding protein regucalcin is highly conserved in vertebrate species. Int J Mol Med 6: 191-196, 2000.

5. Yamaguchi M: Role of regucalcin in calcium signaling. Life Sci 66: 1769-1780, 2000.

6. Yamaguchi M: Role of regucalcin in maintaining cell homeostasis and function (Review). Int J Mol Med 15: 371-389, 2005.

7. Yamaguchi $\mathrm{M}$ : Regucalcin and cell regulation: Role as a suppressor in cell signaling. Mol Cell Biochem 353: 101-137, 2011.

8. Shimokawa N, Matsuda Y and Yamaguchi M: Genomic cloning and chromosomal assignment of rat regucalcin gene. Mol Cell Biochem 151: 157-163, 1995.

9. Thiselton DL, McDowall J, Brandau O, Ramser J, d'Esposito F, Bhattacharya SS, Ross MT, Hardcastle AJ and Meindl A: An integrated, functionally annotated gene map of the DXS8026-ELK1 interval on human Xp11.3-Xp11.23: Potential hotspot for neurogenetic disorders. Genomics 79: 560-572, 2002.

10. Yamaguchi $M$, Makino $R$ and Shimokawa N: The 5 ' end sequences and exon organization in rat regucalcin gene. Mol Cell Biochem 165: 145-150, 1996

11. Yamaguchi M: Hormonal regulation of regucalcin gene expression: Involvement in cell metabolism. Horm Stud 1: 1, 2013.

12. Yamaguchi M: The transcriptional regulation of regucalcin gene expression. Mol Cell Biochem 346: 147-171, 2011.
13. Yamaguchi M: Role of regucalcin in cell nuclear regulation: Involvement as a transcription factor. Cell Tissue Res 354: 331-341, 2013.

14. Yamaguchi M: Suppressive role of regucalcin in liver cell proliferation: Involvement in carcinogenesis. Cell Prolif 46: 243-253, 2013.

15. Yamaguchi M: The anti-apoptotic effect of regucalcin is mediated through multisignaling pathways. Apoptosis 18: 1145-1153, 2013.

16. Yamaguchi M: Regucalcin and metabolic disorder: Osteoporosis and hyperlipidemia are induced in regucalcin transgenic rats. Mol Cell Biochem 327: 53-63, 2010.

17. Yamaguchi $\mathrm{M}$ and Murata T: Involvement of regucalcin in lipid metabolism and diabetes. Metabolism 62: 1045-1051, 2013.

18. Yamaguchi M: Regucalcin as a potential biomarker for metabolic and neuronal diseases. Mol Cell Biochem 391: 157-166, 2014

19. Yamaguchi M: Involvement of regucalcin as a suppressor protein in human carcinogenesis: Insight into the gene therapy. J Cancer Res Clin Oncol 141: 1333-1341, 2015.

20. Nakagawa T, Sawada N and Yamaguchi M: Overexpression of regucalcin suppresses cell proliferation of cloned normal rat kidney proximal tubular epithelial NRK52E cells. Int J Mol Med 16: 637-643, 2005.

21. Yamaguchi M and Daimon Y: Overexpression of regucalcin suppresses cell proliferation in cloned rat hepatoma H4-II-E cells: Involvement of intracellular signaling factors and cell cycle-related genes. J Cell Biochem 95: 1169-1177, 2005.

22. Tsurusaki Y and Yamaguchi M: Role of regucalcin in liver nuclear function: Binding of regucalcin to nuclear protein or DNA and modulation of tumor-related gene expression. Int J Mol Med 14: 277-281, 2004.

23. Tsurusaki Y and Yamaguchi M: Overexpression of regucalcin modulates tumor-related gene expression in cloned rat hepatoma H4-II-E cells. J Cell Biochem 90: 619-626, 2003.

24. Misawa H, Inagaki S and Yamaguchi M: Suppression of cell proliferation and deoxyribonucleic acid synthesis in the cloned rat hepatoma H4-II-E cells overexpressing regucalcin. J Cell Biochem 84: 143-149, 2001.

25. Murata T and Yamaguchi M: Alternatively spliced variants of the regucalcin gene in various human normal and tumor tissues. Int J Mol Med 34: 1141-1146, 2014.

26. Yamaguchi M, Osuka S, Weitzmann MN, El-Rayes BF, Shoji M and Murata T: Prolonged survival in pancreatic cancer patients with increased regucalcin gene expression: Overexpression of regucalcin suppresses the proliferation in human pancreatic cancer MIA PaCa-2 cells in vitro. Int J Oncol 48: 1955-1964, 2016.

27. Yamaguchi M, Osuka S, Weitzmann MN, Shoji M and Murata T: Increased regucalcin gene expression extends survival in breast cancer patients: Overexpression of regucalcin suppresses the proliferation and metastatic bone activity in MDA-MB-231 human breast cancer cells in vitro. Int J Oncol 49: 812-822, 2016.

28. Yamaguchi M, Osuka S, Weitzmann MN, El-Rayes BF, Shoji M and Murata T: Prolonged survival in hepatocarcinoma patients with increased regucalcin gene expression: HepG2 cell proliferation is suppressed by overexpression of regucalcin in vitro. Int J Oncol 49: 1686-1694, 2016.

29. Yamaguchi M, Osuka S, Shoji M, Weitzmann MN and Murata T: Survival of lung cancer patients is prolonged with higher regucalcin gene expression: Suppressed proliferation of lung adenocarcinoma A549 cells in vitro. Mol Cell Biochem 430: 37-46, 2017.

30. Yamaguchi $\mathbf{M}$ and Isogai $M$ : Tissue concentration of calcium-binding protein regucalcin in rats by enzyme-linked immunoadsorbent assay. Mol Cell Biochem 122: 65-68, 1993.

31. Knowles BB, Howe CC and Aden DP: Human hepatocellular carcinoma cell lines secrete the major plasma proteins and hepatitis B surface antigen. Science 209: 497-499, 1980.

32. Ao L, Guo Y, Song X, Guan Q, Zheng W, Zhang J, Huang H, Zou Y, Guo Z and Wang X: Evaluating hepatocellular carcinoma cell lines for tumour samples using within-sample relative expression orderings of genes. Liver Int 37: 1688-1696, 2017.

33. Fang Z, Tang Y, Fang J, Zhou Z, Xing Z, Guo Z, Guo X, Wang W, Jiao W, Xu Z, et al: See comment in PubMed Commons belowSimvastatin inhibits renal cancer cell growth and metastasis via AKT/mTOR, ERK and JAK2/STAT3 pathway. PLoS One 8: e62823, 2013.

34. Lee ZH, Kwack K, Kim KK, Lee SH and Kim HH: Activation of c-Jun N-terminal kinase and activator protein 1 by receptor activator of nuclear factor kappaB. Mol Pharmacol 58: 1536-1545, 2000. 
35. Cano-Abad MF, Villarroya M, García AG, Gabilan NH and López MG: Calcium entry through L-type calcium channels causes mitochondrial disruption and chromaffin cell death. J Biol Chem 276: 39695-39704, 2001.

36. Chen S, Wang Y, Ruan W, Wang X and Pan C: Reversing multidrug resistance in hepatocellular carcinoma cells by inhibiting extracellular signal-regulated kinase/mitogen-activated protein kinase signaling pathway activity. Oncol Lett 8: 2333-2339, 2014.

37. Chen QW, Edvinsson L and Xu CB: Role of ERK/MAPK in endothelin receptor signaling in human aortic smooth muscle cells. BMC Cell Biol 10: 52, 2009.

38. Serrano-Nascimento C, da Silva Teixeira S, Nicola JP, Nachbar RT, Masini-Repiso AM and Nunes MT: The acute inhibitory effect of iodide excess on sodium/iodide symporter expression and activity involves the PI3K/Akt signaling pathway. Endocrinology 155: 1145-1156, 2014.
39. Palangat M, Grass JA, Langelier MF, Coulombe B and Landick R: The RPB2 flap loop of human RNA polymerase II is dispensable for transcription initiation and elongation. Mol Cell Biol 31: 3312-3325, 2011.

40. Tang SC and Chen YC: Novel therapeutic targets for pancreatic cancer. World J Gastroenterol 20: 10825-10844, 2014.

41. Yamaguchi M, Mori S and Kato S: Calcium-binding protein regucalcin is an activator of $\left(\mathrm{Ca}^{2+}-\mathrm{Mg}^{2+}\right)$-adenosine triphosphatase in the plasma membranes of rat liver. Chem Pharm Bull (Tokyo) 36: 3532-3539, 1988.

42. Yamaguchi M and Murata T: Suppressive effects of exogenous regucalcin on the proliferation of human pancreatic cancer MIA PaCa-2 cells in vitro. Int J Mol Med 35: 1773-1778, 2015.

43. Yamaguchi $M$ and Murata T: Exogenous regucalcin suppresses the proliferation of human breast cancer MDA-MB-231 bone metastatic cells in vitro. Mol Med Rep 12: 7801-7805, 2015. 